\title{
Collineation groups which are sharply transitive on an oval
}

\section{P.B. Kirkpatrick}

\begin{abstract}
Let $G$ be a group of collineations in a projective plane $\Pi$ of order $n$. Suppose that one of the point orbits of $G$ is an oval $\stackrel{C}{=}$ of $\Pi$, and that $G$ acts regularly on this orbit. We prove that $G$ fixes a non-incident point-line pair if either $n$ is even, or $n$ is odd and $G$ is abelian, or $n \neq 11,23,59$ is odd and $\stackrel{C}{=}$ is a pseudo-conic. It is then easy to deduce information about the lengths of the other orbits of $G$, and about the structure of $G$ as an abstract group.
\end{abstract}

\section{Introduction}

General results on the relations between the (point and line) orbits of a collineation group in a finite projective plane have been obtained by, for example, Dembowski [6], Foulser and Sandler [8], and Piper [16]. These results depend on the fact that the orbits form a tactical decomposition of the plane. Parker [15], Hughes [12], and Dembowski [6] proved independently that the number of point orbits is equal to the number of line orbits.

Let $\Pi$ be a finite projective plane of order $n$. An oval of $\Pi$ is a set of $n+I$ points in $\Pi$ no three of which are collinear. The elementary properties of ovals are described in Qvist [17] and Dembowski [5]. If $G$ is a group of collineations of $I$ and one of the point orbits of $G$ is an oval $\underline{\underline{C}}$ of $\Pi$, then also one of the line orbits of $G$ consists of the $n+1$ tangents of $\underline{C}$, and each of the remaining point (line) orbits either consists entirely of exterior points (chords) or consists entirely of interior points (non-secants).

Received 24 April 1974. 
By making further assumptions about the way $G$ acts on the oval $\underline{\underline{c}}$, about the geometric structure of $\underline{\underline{C}}$, or about the structure of $G$ as an abstract group, we might hope to obtain more detailed descriptions of the remaining orbits of $G$.

Only the identity collineation fixes every point of the oval $\underline{\underline{c}}$, that is the collineation group $G$ (with orbit $\underline{\underline{C}}$ ) acts faithfully on $\underline{\underline{C}}$; we say that $G$ acts regularly or sharply tronsitively on $\underline{\underline{C}}$ if it is transitive on the points of $\underline{\underline{C}}$ and no non-trivial collineation in $G$ fixes a point of $\underline{\underline{C}}$. If $G$ acts regularly on $\underline{\underline{\mathrm{C}}}$ then $|G|=n+1$, and we shall then call $(G, \underline{\underline{C}})$ a sharply transitive oval. Singer's Theorem [18] guarantees the existence of sharply transitive ovals in every finite desarguesian plane, the ovals being conics and the groups cyclic.

An oval is a pseudo-conic in the sense of Ostrom [14] if it is the set of all absolute points of a polarity of $\pi$.

The results proved in this paper will imply the following:

THEOREM. If $(G, \underline{\underline{C}})$ is a sharply transitive oval in a finite projective plane of order $n$, then $G$ fixes a non-incident point - line pair provided that either

(i) $n$ is even, or

(ii) $n$ is odd and $G$ is abelian, or

(iii) $n \neq 11,23,59$ is odd and $\mathrm{C}$ is a pseudo-conic.

\section{Assumed results}

We shall assume the following theorems from the theory of finite projective planes and the theory of finite groups. Dembowski [5] or Hughes and Piper [13] is suggested as a general reference.

RESULT 1 (Baer [2]). If $\theta$ is an involutory collineation of a finite projective plane of order $n$, then either $\theta$ is an elation and $n$ is even, or $\theta$ is an homology and $n$ is odd, or the fixed points and lines of $\theta$ form a subplane of order $\sqrt{n}$.

RESULT 2 (Baer [1]). Every polarity of a finite projective plane has absolute points.

RESULT 3 (Parker [15], Hughes [12], Dembowski [6]). The number of 
point orbits, of any collineation group of a finite projective plane, is equal to the number of its line orbits.

RESULT 4 (Hering [11], Dembowski [5], p. 179). Let $\Gamma$ be an abelian 2-group of collineations of a projective plane of order $n \equiv 3(\bmod 4)$.

(a) If $|\Gamma|>2$ and $\Gamma$ is elementary abelian, then $|\Gamma|=4$ and the fixed points and lines of $\Gamma$ are the vertices and sides of a triangle.

(b) If $\Gamma$ is not elementary then $\Gamma$ fixes exactly one point and exactly one line, and the point does not lie on the line.

RESULT 5 (Hering [11]). If $\Gamma$ is a 2-group of collineations of a projective plane of order $n \equiv 3(\bmod 4)$ then $\Gamma$ is cyclic, dihedral, semi-dihedral, or a generalized quaternion group.

RESULT 6 (Piper [16]). Let $\Gamma$ be an abelian collineation group of order $N$ in a projective plane of order $n$, and suppose $\Gamma$ has exactly one point orbit of length $N$. Then either the fixed substructure of $\Gamma$ is a line and at least three points on the line; or it is a point and at least three lines through the point; or $N=n^{2}+n+1, n^{2}, n^{2}-1$, $n^{2}-\sqrt{n}, n(n-1),(n-1)^{2}$, or $(n-\sqrt{n+1})^{2} ;$ or $N=9$ and $n=4$.

RESULT 7 (see Hall [10]). Let $G$ be a finite group. If $G$ is soluble then $G$ has an elementary abelian characteristic subgroup. If $G$ is a $p$-group, for some prime $p$, then $G$ has a non-trivial centre.

RESULT 8 (see Wielandt [19]). Suppose $G$ is a permutation group on a finite set $S$, and $P \in S$. Then

$$
|G|=\left|G_{P}\right| \cdot\left|P^{G}\right|
$$

where $G_{P}$ is the stabilizer in $G$ of $P$ and $P^{G}$ is the orbit of $G$ containing $P$. Also, $G$ permutes the orbits of any normal subgroup $H$ of $G$; in particular, $G$ permutes the fixed points of $H$. Finally, if $G$ is abelian and transitive on $S$ then $G$ is sharply transitive on $S$.

RESULT 9 (see Wielandt [19]). If $G$ is a permutation group on a finite set $S$, and if $\chi(g)$ denotes the number of elements of $S$ fixed by $g \in G$, then the number $t$ of orbits of $G$ is given by: 


$$
\sum_{g \in G} x(g)=t|G|
$$

RESULT 10 (Frobenius' Theorem, see Hall [10], p. 292). The kernel of any Frobenius group $G$ is a normal subgroup of $G$.

RESULT 11 (Feit and Thompson [7]). Every group of odd order is soluble.

RESULT 12 (Burnside [4]). If a finite group $G$ has cyclic Sylow 2-subgroups then $G$ has a normal 2-complement.

RESULT 13 (Brauer and Suzuki [3]). If a finite group $G$ has generalized quaternion Sylow 2-subgroups then $G / O(G)$ has a non-trivial centre, where $O(G)$ denotes the largest normal subgroup of odd order in $G$.

RESULT 14 (see Gorenstein [9], pp. 260-265). Let $G$ be a finite simple group whose Sylow 2-subgroups are either dihedral or semi-dihedral. Then $G$ has only one conjugacy class of involutions.

\section{Sharply transitive ovals of even order}

If $(G, \underline{\underline{C}})$ is a sharply transitive oval in a projective plane II of even order $n$, then $G$ certainly fixes the knot $F$ (point of concurrency of the $n+1$ tangents to $\underline{\underline{C}}$ ), since every collineation which maps $\underline{\underline{C}}$ to itself fixes $F$. Also, no non-trivial element of $G$ fixes a point $X \neq F$, since every line through $F$ is tangent to $\underline{\underline{C}}$ and $G$ acts regularly on the tangents of $\underline{\underline{C}}$. Thus every point orbit of $G$, apart from $\{F\}$, has length $n+1$; and $G$ has exactly $n+1$ point orbits. It follows (Result 3 ) that $G$ has exactly $n+1$ line orbits.

Since any line orbit of length less than $n+1$ has length at most $\frac{1}{3}(n+1)$, simple counting shows that $G$ must have $n$ line orbits of length $n+1$ and one fixed line. We have proved:

THEOREM 1. If $(G, \underline{\underline{C}})$ is a sharply transitive oval in a projective plane of even order $n$, then $G$ fixes exactly one point and one line, the point does not lie on the line, and all other orbits of $G$ have length $n+1$. 


\section{Abelian sharply transitive ovals of odd order}

In this section we prove:

THEOREM 2. Let $(G, \underline{\underline{C}})$ be a sharply transitive oval in a projective plane $I$ of odd order $n$, and suppose that $G$ is abelian. Then the involutions of $G$ are homologies, and $G$ fixes the centre and axis of every involutory homology in $G$.

Proof. Choose an involution $\theta$ in $G$. If $\theta$ is an homology then, since $\langle\theta\rangle \triangleleft G$, the whole group $G$ must fix the centre and axis of $\theta$.

Suppose now that $\theta$ is not an homology. Then the fixed points and lines of $\theta$ form a subplane $\Lambda$ of order $\sqrt{n}$ (Result 1). Choose any point $Q$ of $\underline{\underline{\mathrm{C}}}$, let $R=Q^{\theta}$, let $q, r$ be the tangents at $Q, R$ respectively, and let $P=q \cap r$. Then $G_{P}=\langle\theta\rangle$ and so $\left|P^{G}\right|=\frac{1}{2}(n+I)$, which means that $G$ induces on $\Lambda$ an abelian collineation group $H$ of order $\frac{1}{2}(n+1)$.

The group $H$ has at least one point orbit of length $h=|H|=\frac{1}{2}(n+1)$ and, since $\Lambda$ has order $\sqrt{n}$, at most two such point orbits. If there is exactly one, Result 6 implies that the fixed substructure of $H$ consists either of a line and at least three points on the line, or of a point and at least three lines through the point. (The other alternatives, namely various relations between $h$ and $\sqrt{n}$, are easily seen to be impossible.)

If the fixed substructure is a line and at least three points on it, then the line is a non-secant of $\subseteq$ and the fixed points are interior to $\underline{\underline{\mathrm{C}}}$. The fixed points determine at least three distinct chord orbits of length $\frac{1}{2}(n+1)$ for $G$, and these orbits determine at least three distinct involutions in $G$. By Result 4 these involutions generate a group of order 4 whose fixed points are the vertices of a triangle. The alternative (dual) case similarly gives rise to a contradiction.

We assume therefore that $H$ has two point orbits of length $h$, and let $m=\sqrt{n}$, so that $h=\frac{1}{2}\left(m^{2}+1\right)$ and $m$ is the order of $\Lambda$. Piper ([16], p. 331) remarks that simple calculations show that in such a case there is a subplane of $\Lambda$ whose points form a third point orbit for $H$, and that $B$ has only three point orbits. In our situation, this third 
orbit must have length $m\left(=m^{2}+m+1-2 h\right)$, which is impossible since $|H|=\frac{1}{2}\left(m^{2}+1\right)$.

To establish Piper's assertion, let $X^{H}$ be a point orbit of length less than $h$. Then $\left|H_{X}\right| \neq I$ and $H_{X}$ fixes every point of $X^{H}$ (Result 8). Unless $X^{H}$ is a single point, a set of collinear points, or a triangle, the fixed points of $H_{X}$ form an invariant subplane $\Lambda_{0}$ of $\Lambda$ (with respect to $H$ ). The first three possibilities are easily ruled out using the fact that $B$ has two point orbits of length $h$. Now any line of $\Lambda_{0}$ contains at least one point from the union of the two $h$-orbits, and the lines of $\Lambda_{0}$ through the points of a given $h$-orbit all belong to the same line orbit. So $\Lambda_{0}$ contains at most two line orbits of $H$; in fact $\Lambda_{0}$ can contain only one line orbit, since the orbits have odd length (dividing $\frac{1}{2}\left(m^{2}+1\right)$ ) and the number of lines in $\Lambda_{0}$ is odd. Thus $\Lambda_{0}$ contains only one point orbit (Result 3); indeed, every invariant proper subplane contains only one point orbit. It follows that every point $Y$ in $\Lambda \Lambda_{0}$ which lies on a line 1 of $\Lambda_{0}$ belongs to an $h$-orbit $\left(H_{Y}\right.$ fixes 1 and so fixes every line of $\left.\Lambda_{0}\right)$. The possibility that the set of such points exhausts the two $h$-orbits is easily excluded by counting. Thus if $k$ is the order of $\Lambda_{0}$ then

$$
\left(k^{2}+k+1\right)(m-k)=|H|=\frac{1}{2}\left(m^{2}+1\right),
$$

and $\left|H_{X}\right|=m-k$. Now suppose $\phi \in H_{X}$ and $\phi \neq 1$; then, since each invariant proper subplane (for $H$ ) contains only one point orbit of $H$, $\phi$ fixes no point of $\Lambda \Lambda_{0}$. So $H_{X}$ acts semi-regularly on the points of $\Lambda \Lambda_{0}$, and therefore every invariant proper subplane other than $\Lambda_{0}$ contains at least $m-k$ points. But

$$
(m-k)+k^{2}+k+1>m\left(=m^{2}+m+1-2 h\right),
$$

that is $H$ leaves only one proper subplane invariant. So $H$ has exactly three point orbits. 
This completes the proof of Theorem 2. We note that the intersections with a fixed line, of the chords of $\stackrel{C}{=}$ passing through a fixed point not on that line, form a point orbit of length $\frac{1}{2}(n+1)$. for $G$, and that the remaining points on these chords split into $\frac{1}{2}(n-1)$ orbits of length $n+1$, plus the fixed point. A dual assertion can of course be made about line orbits.

\section{Sharply transitive pseudo-conics of odd order}

Let $(G, \underline{\underline{C}}$ ) be a sharply transitive pseudo-conic (in a projective plane $\pi$ of odd order $n$ ), with associated polarity $\alpha$. Then every collineation $\phi$ in $G$ commutes with $\alpha$ and so $\alpha$ induces a polarity on the incidence structure, formed by the fixed points and lines of $\phi$. If $\phi \neq 1$ this structure cannot be a subplane of $\Pi$, since $\phi$ fixes no point of $\mathrm{C}$ and every polarity of a finite projective plane has absolute points (Result 2). Thus the involutions of $G$ are homologies.

Consider any $\psi$ in $G$ which has prime order $p$ and more than one fixed point, say $\psi$ fixes (at least) the points $A$ and $B$. Now $A B$ cannot be an absolute line, and so $C=A^{\alpha} \cap B^{\alpha}$ is not on $A B$. But $\psi$ fixes $C$ and therefore, since the fixed points and lines form a closed substructure which is not a subplane, all further fixed points of $\psi$ lie on one only of the lines $A B, B C, C A$, say on $A B$. By considering the action of $\psi$ on the points of $B C$, we deduce that $p=2$. It follows that if a non-trivial collineation in $G$ fixes more than one point, then its order is a power of 2 .

Now every collineation of prime order in $G$ fixes at least one point, since $\left(|G|, n^{2}+n+1\right)=1$. So every collineation in $G$ whose order is not a power of 2 fixes exactly one point.

\section{If $X$ in $G$ has order 4 and $X$ fixes more than one point,} consider the involution $\chi^{2}$. The centre $A$ and axis $a$ of the homology $\chi^{2}$ are fixed by $\chi$, and all further fixed points of $\chi$ lie on $a$. Suppose $X$ fixes a point $B$ on $a$, and consider the orbits of the group $\langle\chi\rangle$ acting on the points of $A B$ : these are $\{A\},\{B\}$ and further orbits all of length 4 , so that $n-1 \equiv 0(\bmod 4)$, contradicting $n+1 \equiv 0(\bmod 4)$. 
We have proved:

LEMMA. If $(G, \underline{\underline{C}})$ is a sharply transitive pseudo-conic in a projective plane of odd order, then the involutions of $G$ are homologies, and every other non-trivial collineation in $G$ fixes exactly one point.

This lemma will be very useful in the proof of our main result:

THEOREM 3. Suppose $(G, \underline{\underline{C}})$ is a sharply transitive pseudo-conic in a projective plane II of odd order $n \neq 3,11,23,59$. Then $G$ fixes exactly one point and exactly one line, and the point does not lie on the line.

Proof. We note first that it suffices to prove that $G$ fixes exactly one point, since $G$ then fixes the polar line of this point, and no other line; and the fixed point does not lie on its polar line since $G$ acts

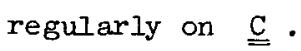

Let $K$ be a non-trivial subnormal subgroup of $G$ such that $K$ is simple. $K$ always exists, and $K=G$ if $G$ is simple. The involutions in $K$ are homologies (Lemma), and they form at most one conjugacy class of $K$ (Results 5, 7, 12, 13, 14). Furthermore, no two involutory homologies in $G$ have the same centre (or the same axis) since the action of such an homology on the oval $\stackrel{C}{=}$ is fully determined by the chords through its centre: it interchanges the two points of $\stackrel{\mathrm{C}}{=}$ on each such chord. Thus the centres of the involutory homologies in $K$ form a point orbit of $K$ whose length equals the number of involutions in $K$.

If $K$ has odd order, then $K$ fixes exactly one point (Results 7,11 , Lemma), and this point is the unique fixed point of $G$. We assume therefore that $K$ has even order.

Any $S_{2}$-subgroup (Sylow 2-subgroup) $S$ of $K$ has a non-trivial centre $Z(S)$. Let $\alpha$ be an involutory homology in $Z(S)$, let $A$ be the centre and $a$ the axis of $\alpha$. Then $K_{A}=K_{a}=c_{K}(\alpha)$, the centralizer in $K$ of $\alpha$; also $K_{A} \supseteq S$, and we have

$$
k=2^{m} r_{c},
$$

if $|K|=k,|S|=2^{m},\left|K_{A}\right|=2^{m} r$ and $c=\left|A^{K}\right|$ is the number of 
involutions in $K$.

Let $\phi$ in $K$ have odd prime order $p$, and fixed point $F$. Any $S_{p}$-şubgroup $P$ of $K$ which contains $\phi$ fixes $F$, since $\left(n^{2}+n+1, p\right)=1$. If $\left|K_{F}\right|$ were odd, then $K$ would act as a Frobenius group on the points of $F^{K}$, that is $K$ would have a proper non-trivial normal subgroup (Result 10), contradicting the simplicity of $K$. So $\left|K_{F}\right|$ is even, that is $F$ is either the centre or lies on the axis of some involutory homology in $K$. If $F$ is a centre then $F \in A^{K}$; while if $F$ is not a centre then $\phi$ does not commute with any of the involutory homologies whose axis contains $F$, and so $F$ lies on at least two axes. In the latter case, the $S_{2}$-subgroups of $K_{F}$ each contain exactly one involution: otherwise the axes of two commuting involutions would both pass through $F$, which is impossible unless $F$ is the centre of the product of these two involutions. It follows that these $S_{2}$-subgroups of $K_{F}$ have order 2 , since if some $\psi$ of order 4 in $K$ fixed $F$ then $F$ would be the unique fixed point of an $S_{2}$-subgroup of $K$ containing $\psi$, that is $F$ would be the centre of an involutory homology.

If a point $X$ lies on the axes of two involutions $B$ and $\gamma$ in $K$ then $\langle\beta, \gamma\rangle$ fixes $X$ and so either $\langle\beta, \gamma\rangle$ is a 2-group and $X$ is fixed by an involution which commutes with both $\beta$ and $\gamma$, that is $X$ is a centre, or $\langle\beta, \gamma\rangle$ is not a 2-group and $X$ is fixed by some collineation of odd order in $K$.

We have established that, for any point $Y$ fixed by an involution in $K$, either $\left|k_{Y}\right|=2$ or $\left|K_{Y}\right|=2^{m_{r}}$ or $\left|K_{Y}\right|=2 s_{i}$ for some odd $s_{i}>1$ coprime to $r$. Furthermore,

$$
k=2^{m} r s_{1} \ldots s_{t},
$$

where $s_{1}, \ldots, s_{t}$ are the distinct numbers $s_{i}$ so arising; and $s_{1}, \ldots, s_{t}$ are mutually coprime.

ASSUMPTION 1. Let us assume that $K$. contains an element of odd order which fixes no centre, that is $t \geq 1$. 
Denote by $c_{0}$ the number of centres on the axis $a$, and let $F_{i}$ be a point on a such that $\left|K_{F_{i}}\right|=2 s_{i}$. By Result 12, $K_{F_{i}}$ has a normal 2-complement $N$. Since no involution in $K_{F_{i}}$ commutes with an element of odd order in $K_{F_{i}}, N$ acts semiregularly on the set of all axes through $F_{i}$. It follows that there are exactly $s_{i}$ axes through $F_{i}$. But $K$ is transitive on the set of all axes (of involutions in $K$ ) and on the points of $F_{i}^{K}$, so we may use simple counting to deduce that the number of points of $\vec{F}_{i}^{K}$ on $a$ is exactly $2^{m-1} r$, for each $i=1,2, \ldots, t$.

To calculate the number $b$ of orbits of $K$, considered as a permutation group on the $\frac{1}{2} n(n-1)$ interior points of $\underline{\underline{C}}$, we apply Result 9, obtaining

$$
\frac{1}{2} n(n-1)+\frac{1}{2}(n+3) c+k-c-1=b k .
$$

Writing $n+1=h k$, we have

$$
b=\frac{1}{2} h(n+c)-h+1 .
$$

We return to the consideration of the points on an axis $a$. The interior points on $a$ consist of: $c_{0}$ centres, $2^{m-1} r t$ points belonging to orbits $F_{i}^{K}$, and $\frac{1}{2}(n+1)-c_{0}-2^{m-1} r t$ points belonging to orbits of length $\frac{1}{2} k$. The third set determines $\left(\frac{1}{2} k\right)^{-1} c\left[\frac{1}{2}(n+1)-c_{0}-2^{m-1} r t\right]$ orbits, so if $c_{0} \neq 0$ there are this number plus $t+1$ orbits consisting of interior points which lie on at least one axis. Since $k \mid n+l$ and $2^{m-1} r c=\frac{1}{2} k, k$ must divide $2 c c_{0}$. But $c_{0}+1$ is the number of involutions in $K_{A}$, since $c_{0}$ is the number of axes through $A$ and $A$ is a centre. Also $\left|k_{A}\right|=2^{m} r$, so that $c_{0}+1 \leq 2^{m} r_{r} 1$, and $c c_{0}<2^{m} r c=k$. Thus either $k=2 c c_{0}$ or $c_{0}=0$.

ASSUMPTION 2. Assume that $k=2 c c_{0}$. 
Combining this with results obtained above, we deduce that there are exactly $\frac{1}{2} h(n-c)-h+1$ orbits of interior points which lie on no axis, and therefore exactly $\left[\frac{1}{2} h(n-c)-h+1\right] k$ such points. We have now counted all the interior points: $c$ centres, $\sum_{i=1}^{t} k\left(2 s_{i}\right)^{-1}$ points which lie on at least two axes (but are not centres), $\left[\frac{1}{2}(n+1)-c_{0}-2^{m-1} r t\right] c$ points which lie on exactly one axis, and $\left[\frac{1}{2} h(n-c)-h+l\right] k$ points which lie on no axis. Thus

$$
\frac{1}{2} n(n-1)=c+\sum_{i=1}^{t} k\left(2 s_{i}\right)^{-1}+\left[\frac{1}{2}(n+1)-c_{0}-2^{m-1} r t\right] c+\left[\frac{1}{2} h(n-c)-h+1\right] k,
$$

from which we deduce the equation

$$
1=c+\sum_{i=1}^{t} k\left(2 s_{i}\right)^{-1}+\frac{1}{2} k(1-t)
$$

and thence (since each $\left.s_{i} \geq 3\right)$ the inequality $c-1 \geq \frac{1}{2} k\left(\frac{2 t}{3}-1\right)$.

If $t \geq 3$ then $c \geq \frac{1}{2} k+1$ and so (since $c \mid k$ ) $c=k$, that is every element of $K$ is an involution, which is impossible. If $t=1$ then, by $(*), 1=c+k\left(2 s_{1}\right)^{-1}$ which is impossible since $c>1, k>0$ and $s_{1}>0$. So $t=2$ and $c-1 \geq \frac{1}{6} k$, that is $c=\frac{1}{5} k, \frac{1}{4} k, \frac{1}{3} k$ or $\frac{1}{2} k$. Now $c \neq \frac{1}{5} k$ or $\frac{1}{3} k$ since $k$ is even and $c$ is odd; and $c \neq \frac{1}{2} k$ since if $c=\frac{1}{2} k$ then $K$ has a normal 2-complement, contrary to the simplicity of $K$.

Thus $t=2$ and $c=\frac{1}{4} k$, that is $k=4 s_{1} s_{2}$ and so, by (*),

$$
1=2 s_{1}+2 s_{2}-s_{1} s_{2} \text {, }
$$

from which we deduce that $\left\{s_{1}, s_{2}\right\}=\{3,5\}, k=60, c=15$ and $c_{0}=2$. Each of the 15 involutions in $k$ commutes with exactly 2 of the remaining 14 , and the 15 centres of these involutory homologies can be partitioned into 5 disjoint sets $\stackrel{C}{G}_{i}$ of 3 non-collinear points which are the centres of the involutory homologies in an elementary abelian $S_{2}$-subgroup (of order 4 ) of $K$. The 15 centres form a unique point 
orbit $\underline{\underline{0}}$ of length 15 for $K$, the remaining point orbits having length $6,10,30$ or 60 . It follows since $K$ is subnormal in $G$, that $\underline{\underline{0}}^{G .}=\underline{\underline{0}}$ and that $G$ permutes the 5 sets $\stackrel{c}{=}$. Since $\stackrel{0}{\underline{O}}^{G}=\underline{\underline{0}}$ and $K$ (being simple) is generated by its 15 involutions, $K \subseteq G$.

The representation of the simple group $K$ as a permutation group on $W=\left\{\underline{\underline{C}}_{1}, \ldots, \underline{\underline{C}}_{5}\right\}$ is faithful. Let $H$ be the kernel of the representation of $G$ on $W$. Since $H \cap K=1, H \triangleleft G$ and $K \unlhd G$, every element of $H$ commutes with each of the 15 involutory homologies in $K$. It follows readily that $H=1$, so that $G$ is isomorphic to a subgroup of $S_{5}$. If $G \cong S_{5}$ then the normalizer in $G$ of any Sylow 5-subgroup $P$ of $G$ contains an element $\phi$ of order $4 ;$ and $\phi$ must fix the point $X$ fixed by $P$. But $\left|X^{G}\right|=6$ and so $\phi$ must fix at least two points of $X^{G}$, contradicting our Lemma. Thus $G \neq S_{5}$ and therefore $|G|=60$, contradicting $n \neq 59$. We have shown that in all cases equation $(*)$ leads to a contradiction.

Suppose that Assumption 2 is false. Then $c_{0}=0$ and so each $S_{2}$-subgroup $S$ of $K$ contains only one involution (otherwise, consider a pair of commuting involutions in $S$ : the centre of one lies on the axis of the other). If $S$ is cyclic then $K$ has a normal 2-complement and so, since $K$ is simple of even order, $|K|=2$. If $S$ is generalized quaternion then $K / O(K)$ has a non-trivial centre (Result 13), which is impossible. There is no other possibility (Result 5), so $|K|=2$.

Now suppose that Assumption $I$ is false. Then $k=2^{m} r, c=I$ and $K$ contains exactly one involution. But $K$ is simple, so $|K|=2$.

Since either Assumption 1 or Assumption 2 is false, $|K|=2$.

If $K$ is a proper subnormal subgroup of some subnormal subgroup $L$ of $G$ which fixes more than one point, then $L$ consists of involutions and the identity, that is $L$ is elementary abelian. Since $K<L$, $|L|=4$ (Result 4 ), and so $L$ fixes exactly three points, the remaining point orbits of $L$ having length 2 or 4 . Now $G$ does not fix all three fixed points of $L$ since $n \neq 3$, so either $G$ fixes exactly one 
point or else $G$ has exactly one point orbit of length 3 . The latter case is impossible since the representation of $G$ as a permutation group on this orbit would have kernel $L$ of order 4 , and the induced group would be isomorphic to a subgroup of $S_{3}$, contradicting $n \neq 11$ or 23 .

If there is no such $L$ then the centre of the involutory homology in $K$ is the unique fixed point of $G$. This completes the proof of Theorem 3.

COROLLARY. Suppose $(G, \underline{\underline{\mathrm{C}}})$ satisfies the hypotheses of Theorem 3. Then either

(i) $G$ contains only one involution, $G$ has two point (line) orbits of length $\frac{1}{2}(n+1)$, and $n-1$ of length $n+1$; or

(ii) $n \equiv 1(\bmod 4), G$ contains $\frac{1}{2}(n+1)$ conjugate involutions, and $G$ has $n+1$ point (Zine) orbits of length $\frac{1}{2}(n+1)$ and $\frac{1}{2}(n-1)$ of length $n+1$; or

(iii) $n \equiv 3(\bmod 4), G$ contains $\frac{1}{2}(n+1)+1$ involutions in three conjugacy classes, of sizes $1, \frac{1}{4}(n+1)$ and $\frac{1}{4}(n+1)$, and $G$ has two point (line) orbits of length $\frac{1}{4}(n+1), n$ of length $\frac{1}{2}(n+1)$ and $\frac{1}{2}(n-1)$ of length $n+1$.

Proof. If $G$ contains only one involution then the centre of this homology is a fixed point, any point on its axis lies in an orbit of length $\frac{1}{2}(n+1)$, and every other point in an orbit of length $n+1$.

Suppose $n \equiv 1(\bmod 4)$ and $G$ contains more than one involution. Then the centres of these involutions must lie on the fixed line $f$ and the axes must pass through the fixed point $F$. Also, no centre lies on an axis, and no axis is a chord of $\underline{\underline{C}}$. But the chords of $\underline{\underline{C}}$ through $F$ meet $f$ in the points of an orbit of length $\frac{1}{2}(n+1)$, so these are the centres of the involutory homologies in $G$, and the remaining $\frac{1}{2}(n+l)$ points on $f$ are the intersections with $f$ of the axes, and form a single orbit. The assertions of (ii) now follow readily.

Finally consider the case where $n \equiv 3(\bmod 4)$ and $G$ contains more than one involution. If $P$ is the intersection with the fixed line $f$ of a chord of $\mathrm{C}$ through the fixed point $F$, then $\left|P^{G}\right|=\frac{1}{2}(n+1)$ and so $\left|G_{P}\right|=2$ and either $G$ contains an involutory $(F, f)$-homology or $P$ is 
a centre or $P F$ is an axis. The third case is impossible since $G$ acts regularly on $\subseteq$. The second case is also impossible since $\left|P^{G}\right|$ is even, which implies that if $P$ is centre of an involutory homology then its axis is also a chord through $F$. Thus $G$ contains an involutory $(F, f)$-homology $\theta$ and, since $\theta$ is in the kernel of the representation of $G$ on $P^{G}, \theta \in Z(G)$. The centre of any other involution in $G$ lies on $f$ in an orbit of length $\frac{1}{4}(n+1)$. Since the length of every point orbit other than $\{F\}$ is at least $\frac{1}{4}(n+1)$, there are two orbits of centres on $f$, each of length $\frac{1}{4}(n+1)$. The assertions of ( $\left.i i i\right)$ now follow readily.

\section{References}

[1] Reinhold Baer, "Polarities in finite projective planes", Buzl. Amer. Math. Soc. 52 (1946), 77-93.

[2] Reinhold Baer, "Projectivities with fixed points on every line of the plane", Bull. Amer. Math. Soc. 52 (1946), 273-286.

[3] Richard Brauer and Michio Suzuki, "On finite groups of even order whose 2-Sylow group is a quaternion group", Proc. Nat. Acad. Sci. U.S.A. 45 (1959), 1757-1759.

[4] W. Burnside, Theory of groups of finite order, and ed. (Combridge University Press, Cambridge, 1911; reprinted Dover, New York, 1955).

[5] P. Dembowski, Finite geometries (Ergebnisse der Mathematik und ihrer Grenzgebiete, Band 44. Springer-Verlag, Berlin, Heidelberg, New York, 1968).

[6]. Peter Dembowski, "Verallgemeinerungen von Transitivitätsklassen endiicher projektiver Ebenen", Math. Z. 69 (1958), 59-89.

[7] Walter Feit and John G. Thompson, "Solvability of groups of odd order", Pacific J. Math. 13 (1963), 773-1029.

[8] David A. Foulser and Reuben Sandler, "Certain properties of orbits under collineation groups", J. Combinatorial Theory 2 (1967), 546-570. 
[9] Daniel Gorenstein, Finite groups (Harper and Row, New York, Evanston, London, 1968).

[10] Marshall Hall, Jr., The theory of groups (The Macmillan Company, New York, 1959).

[11] Christoph Hering, "Eine Bemerkung über Automorphismengruppen von endlichen projektiven Ebenen und Möbiusebenen", Arch. Math. 18 (1967), 107-110.

[12] D.R. Hughes, "Collineations and generalized incidence matrices", Trans. Amer. Math. Soc. 86 (1957), 284-296.

[13] $D[$ aniel] R. Hughes, F[red] C. Piper, Projective planes (Graduate Texts in Mathematics, 6. Springer-Verlag, New York, Heidelberg, Berlin, 1973).

[14] T.G. Ostrom, "Ovals, dualities, and Desargues's theorem", Canad. J. Math. 7 (1955), 417-431.

[15] E.T. Parker, "On collineations of symmetric designs", Proc. Amer. Math. Soc. 8 (1957), 350-351.

[16] Fred Piper, "The orbit structure of collineation groups of finite projective planes", Math. Z. 103 (1968), 318-332.

[17] B. Qvist, "Some remarks concerning curves of the second degree in a finite plane", Ann. Acad. Sci. Fennicae. Ser. A.I. Math.-Phys. 134 (1952), 1-27.

[18] James Singer, "A theorem in finite projective geometry and some applications to number theory", Trans. Amer. Math. Soc. 43 (1938), 377-385.

[19] Helmut Wielandt, Finite permutation groups (Academic Press, New York and London, 1964).

Department of Pure Mathematics,

University of Sydney,

Sydney,

New South Wales. 